\title{
WEAK $P$-POINTS IN COMPACT CCC $F$-SPACES
}

BY

ALAN DOW

\begin{abstract}
Using a technique due to van Mill we show that each compact ccc $F$-space of weight greater than $2^{\omega}$ contains a weak $P$-point, i.e. a point $x \in X$ such that $x \notin \bar{F}$ for each countable $F \subset X-\{x\}$. We show that, assuming $B F(c)$, each nowhere separable compact $F$-space has a weak $P$-point. We show the existence of points which are not limit points of any countable nowhere dense set in compact $F$-spaces of weight $\boldsymbol{\aleph}_{1}$. We also discuss remote points and points not the limit point of any countable discrete set.
\end{abstract}

Introduction. All spaces considered are completely regular and $X^{*}$ denotes $\beta X-X$. A space $X$ is an $F$-space if each cozero set is $C^{*}$-embedded. A ccc $F$-space is easily seen to be extremally disconnected; the closure of each open set is open. A point $x \in X$ is a weak $P$-point if $x \notin \bar{F}$ for each countable $F \subset X-\{x\}$. Kunen [K] has shown that $\omega^{*}$ has a dense set of weak $P$-points. Jan van Mill then showed that each compact infinite $F$-space of weight $2^{\omega}$ in which nonempty $G_{\delta}$ 's have nonempty interior has weak $P$-points [vM]. He also showed that if there is a ccc nonseparable growth of $\omega$ then he could remove the weight restriction. Subsequently Murray Bell [B] constructed such a growth of $\omega$. Then in [DvM] the author and van Mill extended van Mill's result to "each compact nowhere $\operatorname{ccc} F$-space has weak $P$-points ". It is easy to see that a separable space cannot have weak $P$-points. In $\$ 5$ we give an example of a nonseparable $F$-space in which there are no weak $P$-points. We see, therefore, that we need to assume nowhere separable rather than nonseparable. However in the case of ccc spaces we can consider all nonseparable spaces because such a space contains a nowhere separable open set. We address the open question “do all compact nowhere separable $F$-spaces have weak $P$-points?".

We are able to show that for compact $\operatorname{ccc} F$-spaces of weight greater than $c$ the answer is yes and that assuming $B F(c)$ it is also true for nonseparable compact ccc $F$-spaces of weight $\leqslant c$. We are also able to show that for spaces of weight $\boldsymbol{\aleph}_{1}$ we do not need to assume $B F(c)$, that is, there are points not the limit point of any countable nowhere dense set.

The point $x \in X^{*}$ is called a remote point of $X$ if $x \notin \mathrm{cl}_{\beta x} A$ for each nowhere dense subset of $A$ of $X$. It is known that if $X$ is a nonpseudocompact space with countable $\Pi$-weight then $X$ has a remote point [vD, 1.5], [CS]. In [vDvM] the authors show that not every nonpseudocompact space has remote points but ask if $\omega \times 2^{\omega_{2}}$ has remote points if $\mathrm{CH}$ fails. Our methods enable us to show that under $B F(c)$ each nonpseudocompact ccc space of weight $\leqslant c$ has a remote point.

Received by the editors March 20, 1980 and, in revised form, January 16, 1981. 1980 Mathematics Subject Classification. Primary 54D35; Secondary 54D40

$K e y$ words and phrases. Weak $P$-point, $F$-space, ccc. 
Finally we also show that under some further assumptions on the $\Pi$-character of a ccc $F$-space there are points which are not the limit point of any countable discrete set.

1. Nice filters. Let $X$ be a normal space. A filter base on $X$ is a collection of closed subsets of $X$ which is closed under finite intersections and which does not contain the empty set. The filter generated by the filterbase $\mathscr{F}$ is the collection of $\{A \subset X$ : $A=\bar{A}$ and $\exists F \in \mathscr{F}$ with $F \subset A\}$. Let $X$ be the topological sum of countably many nonempty compact spaces, say $X_{n}(n<\omega)$. In [vM] van Mill defines a filter $\mathscr{F}$ to be nice provided that for each $F \in \mathcal{F}$, the set $\left\{n<\omega: F \cap X_{n}=\varnothing\right\}$ is finite while, in addition, $\cap \mathscr{F}=\varnothing$. We will say that a nice filter $\mathscr{F}$ on $X$ avoids countable sets if for each countable $D \subset X$ there is an $F \in \mathcal{F}$ with $\bar{D} \cap F=\varnothing$. In [vM], Jan van Mill has done most of the work of constructing weak $P$-points.

LemMA 1.1. Let $K$ be a compact ccc F-space and let $\left\{Z_{n}: n \in \omega\right\}$ be disjoint nonempty clopen subsets of $K$. Then if $\mathscr{F}$ is a nice filter on $X=\cup Z_{n}$, there is a point $x \in \cap\left\{\mathrm{cl}_{K} F: F \in \mathscr{F}\right\}$ which is not in the closure of any countable subset of $K \backslash(X \cup\{x\})$. In particular, if $\mathscr{F}$ avoids countable subsets of $X$ then $x$ is a weak $P$-point of $K$.

Due to the length and complexity we will not include this proof. The reader is referred to the proof of Theorem 0.3 in $[\mathbf{v M}]$. One need only observe that the restriction of $\mathscr{F}$ to any infinite subcollection of the $Z_{n}$ 's is again a nice filter on this union and that Bell $[\mathbf{B}]$ has shown the existence of a ccc nowhere separable growth of $\omega$. We then follow the proof of Theorem 0.3 in [vM] verbatim.

Our investigation therefore turns to constructing nice filters on topological sums of compact ccc $F$-spaces which avoid countable sets.

2. Large $F$-spaces. We will first investigate compact ccc $F$-spaces with weight greater than $c$. We begin by stating some results which we will require.

THEOREM 2.1 [BF]. Every infinite complete boolean algebra $B$ contains a free subalgebra $A$ with $|A|=|B|$.

The clopen subsets of a compact ccc $F$-space form a complete boolean algebra so by the above result and Stone's duality theorem [W] we obtain

THEOREM 2.2. Each compact ccc F-space of weight greater than $c$ can be mapped onto $2^{c^{+}}$.

Using Theorem 2.2 we can now prove

THEOREM 2.3. Each compact ccc F-space $X$ of weight greater than c contains weak P-points.

Proof. It follows easily from 2.2 that we can choose countably many disjoint clopen subsets $\left\{Z_{n}: n \in \omega\right\}$ of $X$ each of weight greater than $c$. From Lemma 1.1 we need only construct a nice filter $\mathscr{F}$ on $\cup Z_{n}$ which avoids countable subsets of $\cup Z_{n}$. Our technique is very similar to that used in $[\mathbf{v M}]$. By Theorem 2.2, we can let $g_{n}$ be a continuous surjection from $Z_{n}$ to $2^{c^{+}}$. Note that $2^{c^{+}}$is ccc nowhere separable. For 
each countable $D \subset \cup Z_{n}$, let $D^{\prime}=\cup_{n} g_{n}\left[D \cap Z_{n}\right]$. Choose a cellular family of clopen subsets $\left\{A_{k}^{D}: k \in \omega\right\}$ of $2^{c^{+}}$whose union is dense and $A_{k}^{D} \cap D^{\prime}=\varnothing$ for each $k$. This can be done because $D^{\prime}$ is nowhere dense in $2^{c^{+}}$. We will let $\mathscr{F}$ be the filter generated by $\left\{\cup_{n \in \omega} \cup_{k \leqslant n} g_{n}^{-}\left[A_{k}^{D}\right]: D \in\left[\cup Z_{n}\right]^{\omega}\right\}$.

It is a simple matter to check that $\mathscr{F}$ is a nice filter on $\cup Z_{n}$ and obviously $\mathscr{F}$ avoids countable sets. Let $\left\{D_{j}: 1 \leqslant j \leqslant m\right\}$ be countable subsets of $\cup Z_{n}$. Then for each of the cellular families $\left\{A_{k^{\prime}}^{D_{j}}: k \in \omega\right\}, \cup_{k \in \omega} A_{k^{j}}^{D_{j}}$ is dense in $2^{c^{+}}$. Hence we can recursively choose $k_{j}, 1 \leqslant j \leqslant m$, so that $\bigcap_{j=1}^{m} A_{k_{l}}^{D_{j}} \neq \varnothing$. Let $N=\max \left\{k_{j}\right.$ : $1 \leqslant j \leqslant m\}$ and let $n \geqslant N$. Hence

$$
Z_{n} \cap\left(\bigcup_{k \leqslant n} g_{n}^{-}\left[A_{k}^{D_{1}}\right]\right) \cap \cdots \cap\left(\bigcup_{k \leqslant n} g_{n}^{-}\left[A_{k}^{D_{m}}\right]\right)
$$

contains $g_{n}^{-}\left[\cap_{j=1}^{m} A_{k_{j}}^{D_{j}}\right]$ and is therefore not empty. This proves that $\mathscr{F}$ is a nice filter and completes the proof of the theorem.

REMARK. It is worth noting that any ccc nowhere separable space $Y$ could have taken the place of $2^{c^{+}}$in the above proof so long as one has surjections from each $Z_{n}$ to $Y$. This fact can be used to conclude that many well-known $F$-spaces have weak $P$-points, for instance any ccc nowhere separable space which has countably many disjoint clopen sets which are pairwise homeomorphic.

3. Small $F$-spaces. In the case of small $F$-spaces, that is, spaces of weight less than or equal to $c$, we cannot use the above method because $2^{\kappa}$ for $\kappa \leqslant c$ is separable. The method we use is an attempt to capture within the space the essential idea behind the above method. We only managed to succeed with the aid of the set-theoretic principle $B F(c)$. Let $F$ be the set of all functions from $\omega$ into $\omega$. If $f$ and $g$ belong to $F$, define $g \leqslant f$ provided $\{n \in \omega: g(n)>f(n)\}$ is finite. A subset $G$ of $F$ is bounded if there is an $f \in F$ such that for each $g \in G, g \leqslant f$. $B F(c)$ is equivalent to the statement: each subset of $F$ of cardinality less than $c$ is bounded. $B F(c)$ is known to be consistent with the usual axioms of set theory and follows from MA or even $P(c)$ [R, pp. 82, 88].

To prove our result for small nowhere separable compact ccc $F$-spaces we will first prove a lemma in greater generality than is needed for weak $P$-points.

Lemma 3.1. Assume $B F(c)$. Let $\left\{Z_{n}: n \in \omega\right\}$ be compact ccc spaces of weight less than or equal to $c$ and let $X$ be the topological sum of $\left\{Z_{n}: n \in \omega\right\}$. There is a nice filter $\mathscr{F}$ on $X$ which avoids all nowhere dense subsets of $X$. (Such a filter has been called a remote filter $[\mathbf{v M}]$.)

Proof. Since $X$ is ccc and of weight less than or equal to $c$ there are only $c$ maximal cellular families of regular closed sets. Let $\left\{\left\{A_{n, m}^{\alpha}: n, m \in \omega\right\}: \alpha<c\right\}$ list all maximal cellular families of regular closed sets such that for each $\alpha<c$ and $n \in \omega, A_{n, m}^{\alpha} \subset Z_{n}$ for all $m \in \omega$. Let $\mathscr{D}$ be the set of nowhere dense subsets of $X$. Notice that for each $D \in \mathscr{D}$ there is an $\alpha<c$ such that $\bar{D} \cap\left(\cup_{n, m} A_{n, m}^{\alpha}\right)=\varnothing$.

Our plan is to select, for each $\alpha<c$, a function $h_{\alpha}$ from $\omega$ into $\omega$. We will define our filter $\mathscr{F}$ to be generated by the set of closed sets $\left\{\cup_{n \in \omega} \bigcup_{j \leqslant h_{\alpha}(n)} A_{n, j}^{\alpha}: \alpha<c\right\}$. 
So the idea is to select the $h_{\alpha}$ 's to ensure that this filter is nice. This procedure is actually a simple recursion using $B F(c)$.

Let $h_{0}(n)=n$ for each $n \in \omega$. Suppose we have defined $h_{\gamma}$ for $\gamma<\alpha<c$ such that for any finite sequence $\gamma_{1}<\gamma_{2}<\cdots<\gamma_{k}<\alpha$ there is an $N \in \omega$ such that for $n \geqslant N$,

$$
Z_{n} \cap\left[\bigcup_{j \leqslant h_{\gamma_{1}(n)}} A_{n, j}^{\gamma_{1}}\right] \cap \cdots \cap\left[\bigcup_{j \leqslant h_{\gamma_{k}(n)}} A_{n, j}^{\gamma_{k}}\right] \neq \varnothing .
$$

(The $Z_{n}$ is here only for emphasis.) This is the condition we require to ensure we get a nice filter.

Let us select $h_{\alpha}$. For each $E \in[\alpha]^{<\omega}$ we define a function $g_{E}$ as follows. Let $E$ be the sequence $\gamma_{1}<\gamma_{2}<\cdots<\gamma_{k}$. Let $g_{E}(n)=0$ if $\cap_{i=1}^{k}\left[\bigcup_{j \leqslant h_{\gamma_{i}(n)}} A_{n, j}^{\gamma_{i}}\right]=\varnothing$. Otherwise let $g_{E}(n)$ be the smallest integer $p$ such that

$$
A_{n, p}^{\alpha} \cap \bigcap_{i=1}^{k}\left[\bigcup_{j \leqslant h_{\gamma_{t}(n)}} A_{n, j}^{\gamma_{t}}\right] \neq \varnothing .
$$

Since $\left|\left\{g_{E}: E \in[\alpha]^{<\omega}\right\}\right| \leqslant\left|[\alpha]^{<\omega}\right|<c$, the set $\left\{g_{E}: E \in[\alpha]^{<\omega}\right\}$ is a bounded family if we assume $B F(c)$. Hence we can choose a function $h_{\alpha}$ so that for each $E \in[\alpha]^{<\omega}$ the set $\left\{n: g_{E}(n)>h_{\alpha}(n)\right\}$ is finite.

To see that we have preserved our induction assumption, let $E=\left\{\gamma_{i}: 1 \leqslant i \leqslant k\right.$, $\left.\gamma_{i}<\alpha\right\}$. By assumption, there is an integer $N$ such that for $n \geqslant N$,

$$
Z_{n} \cap \bigcap_{i=1}^{k}\left[\bigcup_{j \leqslant h_{\gamma_{i}(n)}} A_{n, j}^{\gamma_{1}}\right] \neq \varnothing \text {. }
$$

Therefore, for $n \geqslant N$,

$$
A_{n, g_{t}(n)}^{\alpha} \cap \bigcap_{i=1}^{k}\left[\bigcup_{j \leqslant h_{\gamma_{i}(n)}} A_{n, j}^{\gamma_{1}}\right] \neq \varnothing .
$$

By the definition of $h_{\alpha}$, there is an integer $N_{1}$, so that for $n \geqslant N_{1}, h_{\alpha}(n) \geqslant g_{E}(n)$. Therefore, for $n \geqslant \max \left(N, N_{1}\right)$,

$$
Z_{n} \cap \bigcap_{i=1}^{k}\left[\bigcup_{j \leqslant h_{\gamma_{1}(n)}} A_{n, j}^{\gamma_{t}}\right] \cap\left[\bigcup_{j \leqslant h_{\alpha}(n)} A_{n, j}^{\alpha}\right] \neq \varnothing .
$$

This completes the induction.

Let $\widetilde{F}$ be the filter generated by the set $\left\{\cup_{n<\omega} \cup_{j \leqslant h_{\alpha}(n)} A_{n, j}^{\alpha}: \alpha<c\right\}$. Since each $A_{n, j}^{\alpha}$ is closed in $Z_{n}$ and we are only taking a finite union in each $Z_{n}$, $\widetilde{F}$ is indeed a filter of closed sets. It follows easily from the induction that $\mathscr{F}$ is nice. Finally, for each $D \in \mathcal{O}_{2}$, as pointed out above, there is an $\alpha<c$ such that $\bar{D} \cap\left(\cup_{n, m} A_{n, m}^{\alpha}\right)=\varnothing$. This completes the proof.

We can now state the main result of this section.

THEOREM 3.2. Assume BF(c). Each compact ccc nowhere separable F-space of weight less than or equal to $c$ contains weak $P$-points. 
Proof. Let $K$ be such an $F$-space and let $\left\{Z_{n}: n \in \omega\right\}$ be nonempty disjoint clopen subsets of $K$ (recall that $K$ is extremally disconnected). By Lemma 1.1, we need only construct a nice filter $\mathscr{F}$ on $X=\cup_{n} Z_{n}$ which avoids countable sets. From Lemma 3.1, we have a nice filter $\mathscr{F}$ on $X$ which avoids all nowhere dense sets. Since $X$ is nowhere separable we are done.

Our theorem concerning remote points also follows from Lemma 3.1.

THEOREM 3.3. Assume BF(c). Each nonpseudocompact ccc space of weight less than or equal to $c$ has remote points.

Proof. Let $X$ be such a space. Since $X$ is not pseudocompact there is a nonempty zero set $Z$ of $\beta X$ contained in $X^{*}$. Let $Y=\beta X \backslash Z$. Since $X$ is ccc and of weight less than or equal to $c$, so is $Y$. Let $\left\{Z_{n}: n \in \omega\right\}$ be a locally finite collection of disjoint nonempty regular closed subsets of $Y$. Let $\mathscr{F}$ be a remote filter on $\cup Z_{n}$ as constructed in Lemma 3.1. Then any $p \in \cap_{F \in \mathcal{F}} \mathrm{cl}_{\beta X} F$ is a remote point of $Y$ and hence of $X$. To see this, first observe that since each $Z_{n}$ is a regular closed subset of $Y$, there is an $F \in \mathcal{F}$ such that $F \subset$ int $_{Y}\left(\cup Z_{n}\right)$. Since $Y$ is normal, $\mathrm{cl}_{\beta X} F \cap \mathrm{cl}_{\beta X}\left(Y \backslash \cup Z_{n}\right)=\varnothing$. Therefore the only nowhere dense subsets of $X$ we have to worry about are those contained in $\cup Z_{n}$, but $\mathscr{F}$ avoids all such sets.

4. Another technique. In this section we develop another technique of constructing nice filters, which is an adaptation of Eric van Douwen's construction of remote points [vD]; the construction in $[\mathbf{C S}]$ is also similar. The $\Pi$-weight, $\Pi w X$, of a space $X$ is the least cardinality of a $\Pi$-base. A $\Pi$-base is a collection of nonempty open sets such that every nonempty open set of the space contains one from the collection. We construct nice filters in compact ccc $F$-spaces of $\Pi$-weight $\aleph_{1}$ which avoid all countable nowhere dense sets. We cannot hope to avoid all countable sets because it is consistent that all such spaces are separable [T]. We use the same method to construct nice filters which avoid countable discrete sets in compact ccc $F$-spaces with further $\Pi$-weight assumptions.

We first need a result of Efimov.

THEOREM 4.1 [E, p. 260]. A compact ccc F-space $X$ with no isolated points can be represented uniquely as $X=\overline{U_{n \in N} U_{\kappa_{n}}}$, where the $U_{\kappa_{n}}$ are disjoint open and closed sets of homogeneous $\Pi$-weight: that is, $\Pi w V=\Pi w U_{\kappa_{n}}$ for any open $V \subset U_{\kappa_{n}}$, and if $n \neq m$ then $\kappa_{n} \neq \kappa_{m}$.

From the above theorem it follows that when we are considering compact ccc $F$-spaces we may assume they have homogeneous $\Pi$-weight. For the remainder of this section all hypothesized spaces will be compact $\operatorname{ccc} F$-spaces with homogeneous $\Pi$-weight. The $\Pi$-character of a point $x$ of $X$, denoted $\Pi \chi(x, X)$, is the least cardinal of a local $\Pi$-base at $x$.

Lemma 4.2. Let $X$ have $\Pi$-weight $\aleph_{1}$. Then

(i) $\Pi \chi(x, X)=\boldsymbol{\aleph}_{1}$ for all $x \in X$, and

(ii) $\Pi \chi(D, X)=\aleph_{1}$ for any countable nowhere dense set $D$, i.e. given countably many open subsets of $X$ there is a neighborhood of $D$ containing none of them. 
Proof. Recall that we are assuming that the $\Pi$-weight of every open set is $\boldsymbol{\aleph}_{1}$.

(i) Let $x \in X$ and let $\left\{A_{n}: n \in \omega\right\}$ be nonempty clopen subsets of $X$. It suffices to find a neighborhood of $x$ which does not contain any of the $A_{n}$ 's. Since $\Pi w\left(A_{n}\right)=\boldsymbol{\aleph}_{1}$ for each $n \in \omega$, we can begin by finding a clopen set $B_{0}$ such that $B_{0} \subset A_{0}$ and $A_{n} \backslash B_{0} \neq \varnothing$ for all $n \in \omega$. Similarly the set $\left\{A_{n} \backslash B_{0}: n \in \omega\right\}$ is not a $\Pi$-base for any open set, in particular, not for $A_{0} \backslash B_{0}$. We can therefore choose a clopen set $B_{1}$ such that $B_{1} \subset A_{0} \backslash B_{0}$ and $\left(A_{n} \backslash B_{0}\right) \backslash B_{1} \neq \varnothing$ for all $n \in \omega$. Similarly, recursively select clopen sets $B_{2 n}$ and $B_{2 n+1}$ such that

(1) $B_{i} \cap B_{j}=\varnothing$ for $i<j \leqslant 2 n+1$,

(2) $B_{2 n} \cup B_{2 n+1} \subset A_{n}$, and

(3) $A_{k} \backslash\left[\cup_{j=1}^{2 n+1} B_{j}\right] \neq \varnothing$ for all $k \in \omega$.

Therefore both $\bigcup_{j \in \omega} B_{2 j}$ and $V=\overline{\bigcup_{j \in \omega} B_{2 j+1}}$ intersect each $A_{n}$ by (2) and $U \cap V=\varnothing$ because $X$ is extremally disconnected. Hence either $U$ or $X \backslash U$ is the required neighborhood of $x$ because neither set contains any of the $A_{n}$ 's.

(ii) Let $D=\left\{d_{n}: n \in \omega\right\}$ be nowhere dense in $X$ and let $\left\{A_{n}: n \in \omega\right\}$ be clopen subsets of $X$. We again wish to find a neighborhood of $D$ which does not contain any $A_{n}$. One recursively selects nonempty clopen sets $B_{k}$ and $C_{k}$ for $k \in \omega$ such that:

(1) $d_{k} \in B_{k}, \quad B_{k} \cap\left[\cup_{j<k} C_{j}\right]=\varnothing$ and $A_{n} \backslash\left[B_{k} \cup \cup_{j<k}\left(B_{j} \cup C_{j}\right)\right] \neq \varnothing$ for $n \in \omega$; and

(2) $C_{k} \cap \bar{D}=\varnothing, C_{k} \subset A_{k} \backslash\left[\cup_{j \leqslant k} B_{j}\right]$ and $A_{n} \backslash\left[\cup_{j \leqslant k}\left(B_{j} \cup C_{j}\right)\right] \neq \varnothing$ for $n \in \omega$. This recursion can be carried out because $\Pi_{\chi}\left(d_{k}, X\right)=\boldsymbol{\aleph}_{1}$ for each $k \in \omega$, $\Pi w\left(A_{n} \backslash\left[\cup \cup_{j \leqslant k}\left(B_{j} \cup C_{j}\right)\right]\right)=\aleph_{1}$ and because $\bar{D}$ is nowhere dense in $X$. This completes the proof.

THEOREM 4.3. Let $X$ have $\Pi$-weight $\boldsymbol{\aleph}_{1}$. There are points in $X$ which are not in the closure of any countable nowhere dense sets.

Proof. Let $\left\{Z_{n}: n \in \omega\right\}$ be disjoint nonempty clopen subsets of $X$. By Lemma 1.1 we must construct a nice filter on $\cup Z_{n}$ which avoids all countable nowhere dense subsets of $\cup Z_{n}$. For each $n \in \omega$, let $\left\{B_{n, \alpha}: \alpha<\omega_{1}\right\}$ be nonempty clopen subsets of $Z_{n}$ which form a $\Pi$-base for $Z_{n}$. Let $\left.\mathscr{Q}\right)=\left\{D \subset \cup Z_{n}: D\right.$ is countable and nowhere dense $\}$. We will construct a family $\left\{F_{D, n}: D \in \omega, n \in \omega\right\}$ of clopen sets satisfying:

(1) $F_{D, n} \cap \bar{D}=\varnothing$;

(2) for any $\& \subset$ (D) with $1 \leqslant|\&| \leqslant n, \cap_{D \in \&} F_{D . n} \neq \varnothing$; and

(3) $F_{D, n} \subseteq Z_{n}$; and then define $F_{D}=\cup_{n} F_{D, n}$.

Our nice filter will be $\widetilde{\mathcal{F}}$ which has $\left\{F_{D}: D \in \mathrm{Q} D\right\}$ as a filter base.

For each $\left.D \in v^{\prime}\right)$ and $n \in \omega$ we define $\alpha(D, n, 0)=\min \left\{\alpha: B_{n, \alpha} \cap \bar{D}=\varnothing\right\}$. Note that $\alpha(D, n, 0)$ exists because $\bar{D}$ is nowhere dense. By Lemma $4.2(\mathrm{ii})$ there is a clopen neighborhood $U(D, n, 1)$ of $D$ such that $B_{n, \gamma} \backslash U(D, n, 1) \neq \varnothing$ for each $\gamma<\alpha(D, n, 0)$. Let $\alpha(D, n, 1)=\min \{\alpha$ : for each $\gamma \leqslant \alpha(D, n, 0)$ there is a $\xi<\alpha$ with $\left.B_{n, \xi} \subset B_{n, \gamma} \backslash U(D, n, 1)\right\}$.

Then let $K(D, n, 1)=\left\{\xi<\alpha(D, n, 1): B_{n, \xi} \cap U(D, n, 1)=\varnothing\right\}$. Note that $\bar{\cup}\left\{B_{n, \xi}: \xi \in K(D, n, 1)\right\} \cap \bar{D}=\varnothing$ and that, for each $\gamma<\alpha(D, n, 0)$, there is a $\xi \in K(D, n, 1)$ with $B_{n, \xi} \subset B_{n, \gamma}$. Recursively construct for $m<n, U(D, n, m+1)$, clopen neighborhoods of $D$ such that for each $\gamma<\alpha(D, n, m)$,

$$
B_{n, \gamma} \backslash U(D, n, m+1) \neq \varnothing,
$$


ordinals $\alpha(D, n, m+1)=\min \{\alpha$ : for each $\gamma \leqslant \alpha(D, n, m)$ there is a $\xi<\alpha$ with $\left.B_{n, \xi} \subset B_{n, \gamma} \backslash U(D, n, m+1)\right\}$, and sets $K(D, n, m)=\{\xi<\alpha(D, n, m+1)$ : $\left.B_{n, \xi} \cap U(D, n, m+1)=\varnothing\right\}$. Define

$$
F_{D, n}=B_{n, \alpha(D, n, 0)} \cup \bigcup_{m=1}^{n}\left[\overline{\bigcup\left\{B_{n, \xi} ; \xi \in K(D, n, m)\right\}}\right] .
$$

Let us check that this satisfies (2). Let $\mathcal{E}$ be a subfamily of $\mathscr{Q})$ with $1 \leqslant|\mathcal{E}| \leqslant n$. Let $|\mathcal{E}|=e$. With recursion on $j$ pick $E_{j} \in \mathcal{E}-\left\{E_{i}: 0 \leqslant i\right.$ and $\left.i<j\right\}$, for $0 \leqslant j<e$ in such a way that

(4) $\alpha\left(E_{j}, n, j\right) \leqslant \alpha(E, n, j)$ for all $E \in \mathcal{E}-\left\{E_{i}: 0 \leqslant i\right.$ and $\left.i<j\right\}$.

Next define $s(j) \in \omega_{1}$, for $0 \leqslant j<e$ by $s(0)=\alpha\left(E_{0}, n, 0\right)$;

$$
s(j+1)=\min \left\{\xi \in K\left(E_{j+1}, n, j+1\right): B_{n, \xi} \subseteq B_{n, s(j)}\right\} .
$$

This is possible by the definition of $K\left(E_{j}, n, j+1\right)$ and the fact that $s(j)<\alpha\left(E_{j}, n, j+1\right)$ for each $0 \leqslant j<e$ by (4). Since each $B_{n, s(j)} \subset F_{E_{j, n}}$ for $0 \leqslant j<e$, it follows that $\bigcap_{j<e} F_{E_{j, n}} \supseteq B_{n, s(e)} \neq \varnothing$. This completes the proof.

Corollary 4.4. Let $X$ have $\Pi$-weight $\boldsymbol{\aleph}_{1}$ and be nowhere separable. Then $X$ contains weak P-points.

REMARK. An easy adaptation of the proof of Theorem 4.3 can be used to show the following. Let $X$ be a nonpseudocompact space of $\Pi$-weight at most $\boldsymbol{\aleph}_{1}$. Then there is a point $x \in X^{*}$ such that $x \notin \bar{F}$ for any countable nowhere dense $F \subset X$ of $\Pi$-character $\aleph_{1}$.

THEOREM 4.5. Let $\Pi w(X)=\kappa$ be a regular cardinal and suppose that $\Pi \chi(x, X)=\kappa$ for each $x \in X$. Then $X$ contains points which are not the limit point of any countable discrete set.

Proof. If $\Pi w(X)=\omega$, this is shown in [vM]. For larger cardinals $\kappa$, it is simple to show that $\Pi \chi(D, X)=\kappa$ for each countable discrete set $D$ in $X$. Then simply replace $\kappa_{1}$ by $\kappa$ in the proof of 4.3 .

We do not know if it is necessarily true that $\Pi \chi(x, X)=\Pi w(X)$ for each $x \in X$ in a compact ccc $F$-space of homogeneous $\Pi$-weight. We showed that it was true for $\Pi$-weight $\aleph_{1}$ in 4.2 and in the following theorem we show it for separable compact $F$-spaces assuming Martin's axiom for $\sigma$-centred posets, denoted MAS.

THEOREM 4.6 (MAS). Let $X$ be a compact separable F-space. Then $\Pi \chi(x, X)=\Pi w(X)$ for each $x \in X$.

Proof. Let $\Pi w(X)=\kappa$ and note that $\kappa \leqslant c$ since $X$ is separable. Let $S$ be a countable dense subset of $X$ and suppose there is an $x \in X$ with $\Pi \chi(x, X)<\kappa$. Let us first prove the following fact.

FACT 1. Suppose that $x$ is the limit point of a discrete set $D$ and that $\Pi \chi(d, X)>\lambda$ for each $d \in D$. Then $\Pi \chi(x, X)>\lambda$. Indeed, suppose $\left\{A_{\alpha}: \alpha<\lambda\right\}$ is a collection of clopen subsets of $X$. To show that it is not a $\Pi$-base at $x$ we will find a clopen neighborhood of $x$ not containing any $A_{\alpha}$. Since $D$ is discrete, let $D=\left\{d_{n}: n \in \omega\right\}$ and $\left\{V_{n}: n \in \omega\right\}$ be disjoint neighborhoods of the $d_{n}$ 's. Since $\Pi \chi\left(d_{n}, X\right)>\lambda$ for 
each $n$, we can choose a neighborhood $U_{n}$ of $d_{n}$ such that $U_{n} \subset V_{n}$ and, if $A_{\alpha} \cap V_{n} \neq \varnothing$, then $\left(A_{\alpha} \cap V_{n}\right) \backslash U_{n} \neq \varnothing$ for $\alpha<\lambda$. Since $X$ is extremally disconnected and $x \in U=\bar{U}_{n}, U$ is a neighborhood of $x$. Let $\alpha<\lambda$ and suppose that $A_{\alpha} \subset U$. Then there is an $n<\omega$ such that $A_{\alpha} \cap U_{n} \neq \varnothing$ and therefore $A_{\alpha} \cap V_{n} \neq \varnothing$. This is a contradiction because $\left(A_{\alpha} \cap V_{n}\right) \backslash U_{n} \neq \varnothing$ by assumption and $\left(U \backslash U_{n}\right) \cap\left(A_{\alpha} \cap V_{n}\right)=\varnothing$. Therefore Fact 1 is true.

Now suppose that $x \in X$ and $\Pi \chi(x, X)=\lambda<\kappa$. Note that $\{s \in S$ : $\Pi \chi(s, X) \leqslant \lambda\}$ is nowhere dense in $X$ because the union of local $\Pi$-bases of a dense set is a $\Pi$-base for $X$. Therefore we assume $\Pi \chi(s, X)>\lambda$ for each $s \in S$. Our plan now is to find a discrete subset of $S$ which has $x$ as a limit point, which will complete the proof.

Let $\left\{A_{\alpha}: \alpha<\lambda\right\}$ be a $\Pi$-base at $x$ consisting of clopen subsets of $X$. Also let $\left\{B_{\alpha}\right.$ : $\alpha<\kappa\}$ be clopen subsets of $X$ which form a $\Pi$-base for $X$. Recall that we are assuming that $X$ has homogeneous $\Pi$-weight. We can inductively define $B_{\alpha}^{\prime}$ so that $B_{\alpha}^{\prime} \subset B_{\alpha}$ and for each $\xi<\lambda$ and $\gamma_{1}<\cdots<\gamma_{n}<\alpha, B_{\alpha}^{\prime} \nsupseteq A_{\xi} \backslash \cup_{i=1}^{n} B_{\gamma_{i}}^{\prime}$. Therefore $\left\{B_{\alpha}^{\prime}: \alpha<\kappa\right\}$ is a $\Pi$-base such that no finite union of its members contains any $A_{\xi}$. Let $(P, \leqslant)$ be the poset whose members are $\left\{(F, V): F \in[S]^{<\omega}, V\right.$ is a finite union of sets from the $\Pi$-base; and $F \subset V\}$. We will define $(G, W) \leqslant(F, V)$ if $F \subset G$, $V \subset W$ and $G \backslash F \cap V=\varnothing$. To see that $(P, \leqslant)$ is $\sigma$-centred, we simply let $P_{F}=\{(F, V):(F, V) \in P\}$ for each $F \in[S]^{<\omega}$. Let $E_{\alpha}=\left\{(F, V): F \cap A_{\alpha} \neq \varnothing\right\}$ for each $\alpha<\lambda$. It is easy to check that $E_{\alpha}$ is dense in $(P, \leqslant)$. Therefore MAS allows us to choose a generic filter $\mathcal{G}$ such that for each $\alpha<\lambda$ there is an $\left(F_{\alpha}, V_{\alpha}\right) \in \mathcal{G}$ with $\left(F_{\alpha}, V_{\alpha}\right)$ also in $E_{\alpha}$. Define $D=\cup\{F: \exists V$ with $(F, V) \in \mathcal{G}\}$.

First note that $D \cap A_{\alpha} \neq \varnothing$ for each $\alpha<\lambda$ and so $x \in \bar{D}$. All we have to show is that $D$ is discrete. Let $d \in D$ and find $\left(F_{D}, V_{D}\right) \in \mathcal{G}$ with $d \in F_{D}$. By the definition of the partial ordering we see that $D \cap V_{D}=F_{D}$. It follows that $D$ is discrete.

We remark that MA implies that if $X$ is compact, ccc and $\Pi w(X)<c$ then $X$ is separable [T].

COROllaRY 4.7 (MAS). Let $X$ be a compact separable F-space with $\Pi w(X)$ regular; then $X$ contains points not the limit point of any countable discrete set.

Proof. Theorems 4.5 and 4.6.

\section{Example and remarks.}

EXAMPLE. We now give the promised example of a compact nonseparable $F$-space which does not have any weak $P$-points. The author is grateful to the referee and also to Jan van Mill for suggesting this example. Let $\omega_{1}+1$ be the ordinals less than or equal to $\omega_{1}$ endowed with the order topology. Let $E$ be the projective cover of $\omega_{1}+1$ and let $k: E \rightarrow \omega_{1}+1$ be the canonical map. Let $Z=k^{\leftarrow}\left[\left\{\omega_{1}\right\}\right]$ and let $Y=\beta(\omega \times E)$. Observe that $Y-\operatorname{cl}_{Y}(\omega \times Z)$ is locally separable. Let $\Pi$ : $\omega \times Z \rightarrow \omega$ be the projection map and let $\beta \Pi: \beta(\omega \times Z) \rightarrow \beta \omega$ be its Stone extension. Note that $\beta(\omega \times Z)=\operatorname{cl}_{Y}(\omega \times Z)$, as $Y$ is an $F$-space and $\omega \times Z$ is $\sigma$-compact. We can form the adjunction space $M=Y \cup_{\beta I I} \beta \omega$ (see [W, Chapter 10]). Since $\operatorname{cl}_{Y}(\omega \times Z)$ is a $P$-set of $Y$ it is easy to show that $M$ is an $F$-space. Clearly $M$ is not separable and has no weak $P$-points. 
REMARKS. One would naturally conjecture that all compact nowhere separable $F$-spaces contain weak $P$-points. The remaining problem is to remove special set-theoretic assumptions for the case of compact ccc $F$-spaces of weight $\leqslant c$. Murray Bell has observed that if such a space has a $\sigma$ - $n$-linked base for each $n$ then it contains weak $P$-points. One would also like to show that all compact $F$-spaces contain points which are not the limit point of any countable discrete set. Is it true that all compact ccc $F$-spaces of homogeneous $\Pi$-weight also have homogeneous П-character?

\section{REFERENCES}

[B] Murray G. Bell, Compact ccc non-separable spaces of small weight, Topology Proc. (to appear).

[BF] B. Balcar and F. Franek, Independent families on complete Boolean algebras, Trans. Amer. Math. Soc. (to appear).

[CS] S. B. Chae and J. H. Smith, Remote points and G-spaces, Topology Appl. 11 (1981), 243-246.

[vD] Eric K. van Douwen, Remote points, Dissertationes Math. (to appear).

[vDvM] Eric K. van Douwen and Jan van Mill, Spaces without remote points, Pacific J. Math. (to appear).

[DvM] A. Dow and J. van Mill, On nowhere dense ccc P-sets, Proc. Amer. Math. Soc. 80 (1980), 697-700.

[E] B. Efimov, Extremally disconnected compact spaces and absolutes, Trudy Moskov. Mat. Obšč. 23 (1970), 235-276.

[K] Ken Kunen, Weak P-points in $N^{*}$, Proc. Bolyái János Soc. Colloq. on Topology (Budapest, 1978), vol. 2, pp. 741-750.

[vM] Jan van Mill, Weak P-points in compact F-spaces, Topology Proc. 4 (1979), 609-628.

[R] George M. Reed, Set-theoretic topology, Academic Press, New York, 1977.

[T] F. D. Tall, The countable chain condition versus separability-Applications of Martin's axiom, General Topology Appl. 4 (1974), 315-339.

[W] R. C. Walker, The Stone-Čech compactification, Springer-Verlag, New York, 1974.

Department of Mathematics, University of Kansas, Lawrence, Kansas 66045 